\title{
Chloroquine Induces Human Macrophage Killing of Histoplasma capsulatum by Limiting the Availability of Intracellular Iron and Is Therapeutic in a Murine Model of Histoplasmosis
}

\author{
Simon L. Newman, Lisa Gootee, George Brunner, and George S. Deepe, Jr. \\ Department of Medicine, Division of Infectious Diseases, University of Cincinnati College of Medicine, Cincinnati, Ohio 45267
}

\begin{abstract}
We investigated the role of intracellular iron on the capacity of Histoplasma capsulatum (Hc) yeasts to multiply within human macrophages $(\mathrm{M} \phi)$. Coculture of Hc-infected $M \phi$ with the iron chelator deferoxamine suppressed the growth of yeasts in a concentration-dependent manner. The effect of deferoxamine was reversed by iron-saturated transferrin (holotransferrin) but not by iron-free transferrin (apotransferrin). Chloroquine, which prevents release of iron from transferrin by raising endocytic and lysosomal $\mathrm{pH}$, induced human $\mathrm{M} \phi$ to kill Hc. The effect of chloroquine was reversed by iron nitriloacetate, an iron compound that is soluble at neutral to alkaline $\mathrm{pH}$, but not by holotransferrin, which releases iron only in an acidic environment.

Chloroquine (40-120 mg/ kg) given intraperitoneally for 6 d to Hc-infected C57BL/6 mice significantly reduced the growth of $\mathrm{Hc}$ in a dose-dependent manner. At $120 \mathrm{mg} / \mathrm{kg}$ there was a 17- and 15-fold reduction $(P<0.01)$ in $C F U$ in spleens and livers, respectively. The therapeutic effect of chloroquine also correlated with the length of treatment. As little as $\mathbf{2} \mathrm{d}$ of chloroquine therapy $(120 \mathrm{mg} / \mathrm{kg})$, when started at day 5 after infection, reduced CFU in the spleen by $50 \%$. Treatment with chloroquine for $10 \mathrm{~d}$ after a lethal inoculum of $\mathrm{Hc}$ protected six of nine mice; all control mice were dead by day $11(P=0.009)$.

This study demonstrates that: $(a)$ iron is of critical importance to the survival and multiplication of Hc yeasts in human $\mathbf{M} \phi ;(b)$ in vitro, chloroquine induces $M \phi$ killing of Hc yeasts by restricting the availability of intracellular iron; and $(c)$ in vivo, chloroquine significantly reduces the number of organisms in the spleens and livers of Hc-infected mice and can protect mice from a lethal inoculum of Hc yeasts. Thus, chloroquine may be effective in the treatment of active histoplasmosis and also may be useful in preventing relapse of histoplasmosis in patients with acquired immunodeficiency syndromes. ( $J$. Clin. Invest. 1994. 93:1422-1429.) Key words: macrophage • Histoplasma capsulatum • chloroquine • iron • transferrin
\end{abstract}

Address correspondence to Simon L. Newman, Division of Infectious Diseases, University of Cincinnati Medical Center, 231 Bethesda Avenue (ML 560), Cincinnati, OH 45267.

Received for publication 17 August 1993 and in revised form 29 October 1993.

J. Clin. Invest.

(c) The American Society for Clinical Investigation, Inc.

$0021-9738 / 94 / 04 / 1422 / 08 \$ 2.00$

Volume 93, April 1994, 1422-1429

\section{Introduction}

The mechanisms used by Histoplasma capsulatum $(\mathrm{Hc})^{1}$ yeasts to survive and multiply within human macrophages $(\mathbf{M} \phi)$ are poorly understood. Phagocytosis of $\mathrm{Hc}$ yeasts by human monocyte $/ \mathrm{M} \phi$ stimulates both the respiratory burst (1-3) and phagolysosomal fusion (4). Nevertheless, ingested yeasts multiply within $\mathrm{M} \phi$ phagolysosomes (5-7).

Recent studies on the interaction of $\mathrm{Hc}$ yeasts with murine peritoneal $\mathrm{M} \phi(\mathrm{PM})$ suggest that acquisition of intracellular iron may be important for yeast survival. Thus, coculture of Hc-infected murine PM in the presence of the iron chelator deferoxamine suppresses the intracellular growth of yeasts, and this effect is reversed by iron-saturated transferrin (holotransferrin ( 8 ). Furthermore, it has been suggested that one mechanism by which IFN $\gamma$-activated mouse PM (8) and IFN $\gamma$-LPSactivated murine splenic $M \phi(9)$ inhibit the intracellular growth of $\mathrm{Hc}$ is by the restriction of intracellular iron. Likewise, Byrd and Horwitz $(10,11)$ have demonstrated that the intracellular multiplication of Legionella pneumophila in human monocytes is iron dependent. IFN $\gamma$ downregulates monocyte transferrin receptors and, therefore, suppresses intracellular growth of $L$. pneumophila by restricting the availability of iron (9).

Although in conflict with one report (12), we (7) and others (5) have found that human M $\phi$ stimulated with IFN $\gamma$ do not inhibit the intracellular growth of Hc yeasts. Thus, downregulation of transferrin receptors and the concomitant restriction of iron in IFN $\gamma$-activated human $\mathrm{M} \phi$ apparently are not sufficient to suppress the intracellular growth of $\mathrm{Hc}$ yeasts. Since the availability of intracellular iron clearly is required for the intracellular growth of $\mathrm{Hc}$ yeasts in murine $\mathrm{M} \phi$, we sought to determine the role of intracellular iron in regulating the capacity of Hc yeasts to survive and multiply within human $\mathbf{M} \phi$.

\section{Methods}

Reagents. Deferoxamine, chloroquine, holotransferrin, apotransferrin, iron nitriloacetate (FeNTA), and nitriloacetate (NTA) were purchased from Sigma Chemical Co. (St. Louis, MO). FeNTA and its control, NTA, were prepared fresh for each experiment as described (13). NTA was prepared in distilled water and mixed 1:1 with ferric chloride in $1 \mathrm{~N} \mathrm{HCl}$. The $\mathrm{pH}$ of the solution was adjusted to 7.0 with 1 $\mathrm{N} \mathrm{NaOH}$. NTA was prepared in the same manner, but without ferric

1. Abbreviations used in this paper: FeNTA, iron nitriloacetate; HBSA, HBSS containing $0.25 \%$ BSA; Hc, Histoplasma capsulatum; $\mathrm{M} \phi$, macrophage; NTA, nitriloacetate; PM, peritoneal $\mathrm{M} \phi$. 
chloride. FeNTA and NTA were sterile filtered through a $0.22-\mu \mathrm{m}$ filter unit before culturing with $\mathrm{Hc}$-infected $\mathrm{M} \phi$. All other reagents were prepared in RPMI 1640 (Gibco Laboratories, Grand Island, NY) and sterile filtered.

Yeasts. H. capsulatum strain G217B was maintained as described $(1,2)$. Yeasts were grown in $\mathrm{HMM}$ medium $(14)$ at $37^{\circ} \mathrm{C}$ with orbital shaking at $150 \mathrm{rpm}$. After $2 \mathrm{~d}$, they were harvested by centrifugation, washed three times in HBSS containing $20 \mathrm{mM}$ Hepes and $0.25 \%$ BSA (HBSA), and resuspended to $30 \mathrm{ml}$ in HBSA. Large aggregates were removed by centrifugation at $200 \mathrm{~g}$ for $5 \mathrm{~min}$ at $4^{\circ} \mathrm{C}$. The top $2 \mathrm{ml}$ was removed, and the single cell suspension obtained was standardized to 5 $\times 10^{4} / \mathrm{ml}$ in RPMI 1640 containing 5\% heat-inactivated FCS and 10 $\mu \mathrm{g} / \mathrm{ml}$ of gentamicin (Sigma Chemical Co.) (2).

Monocyte preparation and culture. Human peripheral blood monocytes were prepared under sterile conditions by dextran sedimentation and Ficoll-Hypaque centrifugation as described previously (15). The mononuclear cells were washed in HBSS containing $20 \mathrm{mM}$ Hepes and $10 \mu \mathrm{g} / \mathrm{ml}$ gentamicin (Hanks' / Hepes) and suspended to $2-3 \times 10^{6} / \mathrm{ml}$ in Hanks'/Hepes containing $0.1 \%$ autologous serum. $0.1-\mathrm{ml}$ volumes of mononuclear leukocytes were adhered in 96-well tissue culture plates (Corning Medical \& Scientific, Cambridge, MA) for $1 \mathrm{~h}$ at $37^{\circ} \mathrm{C}$ in $5 \% \mathrm{CO}_{2} / 95 \%$ air. The adherent monocytes were washed vigorously with Hank's/Hepes to remove the lymphocytes and then were infected with Hc yeasts.

Alternatively, human monocytes were purified from buffy coats via sequential centrifugation on Ficoll-Hypaque and percoll gradients (both from Pharmacia LKB Biotechnology Inc., Piscataway, NJ). The monocytes were cultured in suspension in Teflon beakers at $1 \times 10^{6}$ / $\mathrm{ml}$ in RPMI 1640 containing $12.5 \%$ human serum and $10 \mu \mathrm{g} / \mathrm{ml}$ of gentamicin. After 4-7 d of culture, $M \phi$ were washed and suspended in HBSA containing $0.3 \mathrm{U} / \mathrm{ml}$ of aprotinin to $6.0 \times 10^{5} / \mathrm{ml}(2,6) .0 .1-\mathrm{ml}$ aliquots of $\mathrm{M} \phi$ were adhered in 96-well tissue culture plates for $1 \mathrm{~h}$ at $37^{\circ} \mathrm{C}$ in $5 \% \mathrm{CO}_{2} / 95 \%$ air. $\mathrm{M} \phi$ monolayers were washed once with RPMI 1640 and then were infected with Hc yeasts as described below.

Quantitation of the growth of Hc yeasts in human monocyte/M $\phi$. The intracellular growth of $\mathrm{Hc}$ yeasts in monocyte $/ \mathrm{M} \phi$ was quantified by the incorporation of $\left[{ }^{3} \mathrm{H}\right]$ leucine (7). Supernatant was removed from the wells of monocyte $/ \mathrm{M} \phi$, and $5 \times 10^{3}$ viable yeasts in $0.1 \mathrm{ml}$ of RPMI 1640 containing 5\% heat-inactivated FCS and $10 \mu \mathrm{g} / \mathrm{ml}$ of gentamicin were added to each well. After allowing $1 \mathrm{~h}$ for phagocytosis of the yeasts, various reagents were added, and the monolayers were cultured for a further $24 \mathrm{~h}$ at $37^{\circ} \mathrm{C}$. The plates then were centrifuged at $2,000 \mathrm{rpm}$, the supernatant was carefully aspirated through a 27 -gauge needle, and $50 \mu \mathrm{l}$ of $\left[{ }^{3} \mathrm{H}\right]$ leucine (sp act $153 \mathrm{Ci} / \mathrm{mmol}$; New England Nuclear, Boston, MA) in sterile water $(1.5 \mu \mathrm{Ci})$ and $5 \mu \mathrm{l}$ of $10 \times$ yeast nitrogen broth (Difco Laboratories Inc., Detroit, MI) were added to each well. After further incubation for $24 \mathrm{~h}$ at $37^{\circ} \mathrm{C}, 50 \mu \mathrm{l}$ of L-leucine and $50 \mu \mathrm{l}$ of sodium hypochlorite were added to each well. The contents of the wells were harvested onto glass fiber filters using an automated harvester (Skatron, Inc., Sterling, VA). The filters were placed into scintillation vials, scintillation cocktail was added, and the vials were counted on a liquid scintillation spectrometer (LS 7000; Beckman Instruments, Inc., Fullerton, CA). Control wells containing $\mathbf{M} \phi$ but no yeasts did not incorporate significant amounts of $\left[{ }^{3} \mathrm{H}\right]$ leucine $(<300 \mathrm{cpm})(7)$.

The range of counts per minute obtained from yeasts multiplying in $\mathrm{M} \phi$ from different donors varied considerably (2,000-200,000 cpm). Therefore, the data are presented as the mean \pm SEM of percent inhibition of growth, defined as $1-(\mathrm{cpm}$ in control $\mathrm{M} \phi / \mathrm{cpm}$ in treated $\mathbf{M} \phi)$ $\times 100$. All experiments were performed at least three times, in triplicate using $\mathbf{M} \phi$ from different donors. None of the agents tested decreased $\mathbf{M} \phi$ viability as determined by trypan blue dye exclusion, nor did they cause a loss of $\mathrm{M} \phi$ from the monolayer.

Quantitation of the intracellular growth of $\mathrm{Hc}$ yeasts by phase-contrast microscopy. 1-ml aliquots of cultured $\mathrm{M} \phi$ were adhered to 12-mm diameter glass coverslips in 24-well culture plates (Costar Corp., Cambridge, MA) for $1 \mathrm{~h}$ at $37^{\circ} \mathrm{C}$ in $5 \% \mathrm{CO}_{2} / 95 \%$ air. The cells were washed, and $1 \times 10^{6}$ viable yeasts were added for $30 \mathrm{~min}$ at $37^{\circ} \mathrm{C}$ to allow for phagocytosis. The cells were washed twice in HBSA and incubated an additional $30 \mathrm{~min}$ to insure that all yeasts were internalized. Two of the cell monolayers were fixed in $2 \%$ glutaraldehyde/ $1 \%$ sucrose in $0.01 \mathrm{M}$ phosphate buffer $/ 0.15 \mathrm{M} \mathrm{NaCl}, \mathrm{pH}$ 7.2. These cells provided a baseline for the percentage of $\mathbf{M} \phi$ initially infected and were designated 1-h infection (6).

The remaining $\mathrm{M} \phi$ were cultured in M199 (Gibco Laboratories) containing $10 \%$ human serum and $10 \mu \mathrm{g} / \mathrm{ml}$ gentamicin or in medium containing chloroquine. After $24 \mathrm{~h}$ of culture at $37^{\circ} \mathrm{C}$, medium was removed, the cells were washed, and the monolayers were fixed in glutaraldehyde. Coverslips were mounted cell side down onto microscope slides. The percentage of $\mathrm{M} \phi$ containing one or more intracellular yeasts and the number of yeasts in 100 infected $\mathrm{M} \phi$ were enumerated by phase-contrast microscopy at a magnification of 1,000 . There was no loss of adherent $M \phi$ during these experiments $(4,6)$.

Mice. 5-wk-old C57BL/6J male mice were purchased from Jackson Laboratories (Bar Harbor, ME). The mice were housed four to a cage and adapted to their environment for $1 \mathrm{wk}$ before infection with Hc yeasts.

Injection of mice with $\mathrm{Hc}$ yeasts and treatment with chloroquine. A single cell suspension of yeasts was prepared as described above. Mice were given an intravenous injection with either a sublethal inoculum ( 1 $\left.\times 10^{6}\right)$ or a lethal inoculum $\left(1.5 \times 10^{7}\right)$ of $\mathrm{Hc}$ yeasts in a total volume of $0.2 \mathrm{ml}$ in RPMI 1640 (16). Chloroquine, prepared in RPMI 1640 and sterile filtered, was injected intraperitoneally in a total volume of $0.2 \mathrm{ml}$.

Organ culture for $\mathrm{Hc}$. Spleens and livers from Hc-infected mice were removed aseptically, homogenized in HBSS, and were diluted serially. $100-\mu 1$ of homogenate was plated onto brain-heart infusion agar ( $2 \% \mathrm{wt} / \mathrm{vol})$ supplemented with $10 \%(\mathrm{vol} / \mathrm{vol})$ defibrinated sheep erythrocytes, $10 \mu \mathrm{g} / \mathrm{ml}$ dextrose, and $0.1 \mu \mathrm{g} / \mathrm{ml}$ cysteine hydrochloride. Plates were incubated at $30^{\circ} \mathrm{C}$, and CFU were counted after 7-10 d (16).

Statistics. The Wilcoxon rank sum test was used for comparison of two groups. Fisher's exact test was used to analyze mouse survival data.

\section{Results}

Deferoxamine inhibits the intracellular growth of $\mathrm{Hc}$ yeasts in human monocytes and cultured $M \phi$. In initial experiments we sought to extend to human $\mathrm{M} \phi$ the observations of Lane et al. (8) that deferoxamine inhibits the intracellular growth of $\mathrm{Hc}$ yeasts in murine PM. Human monocyte and $\mathrm{M} \phi$ monolayers were infected with $5 \times 10^{3} \mathrm{Hc}$ yeasts and then were cultured for $24 \mathrm{~h}$ at $37^{\circ} \mathrm{C}$ in the presence of varying concentrations of deferoxamine. Intracellular growth of yeasts was quantified by the incorporation of $\left[{ }^{3} \mathrm{H}\right]$ leucine as described in Methods. Deferoxamine inhibited the capacity of $\mathrm{Hc}$ yeasts to multiply in tissue culture medium and within human monocytes and $\mathrm{M} \phi$ in a concentration-dependent manner (Fig. 1). The viability of control and deferoxamine-treated $\mathbf{M} \phi$ was $>95 \%$ as determined by trypan blue dye exclusion, and deferoxamine did not cause a loss of $\mathbf{M} \phi$ from the monolayers.

Holotransferrin and FeNTA reverse deferoxamine inhibition of the intracellular growth of Hc yeasts. To demonstrate that deferoxamine suppressed the intracellular growth of $\mathrm{Hc}$ yeasts by chelating iron, $\mathrm{Hc}$ yeast-infected $\mathbf{M} \phi$ were cultured for $24 \mathrm{~h}$ in the presence of $2.5 \mathrm{mM}$ deferoxamine alone or with deferoxamine and increasing concentrations of FeNTA or holotransferrin. Control wells contained infected $\mathrm{M} \phi$ incubated in medium alone or in medium containing NTA or apotransferrin. FeNTA and holotransferrin completely reversed the inhibition of yeast growth by deferoxamine (Fig. 2). In fact, in infected $\mathrm{M} \phi$ incubated with $100 \mu \mathrm{g} / \mathrm{ml}$ of FeNTA or $\geq 5 \mathrm{mg} /$ $\mathrm{ml}$ of holotransferrin, the yeasts grew more rapidly than in 


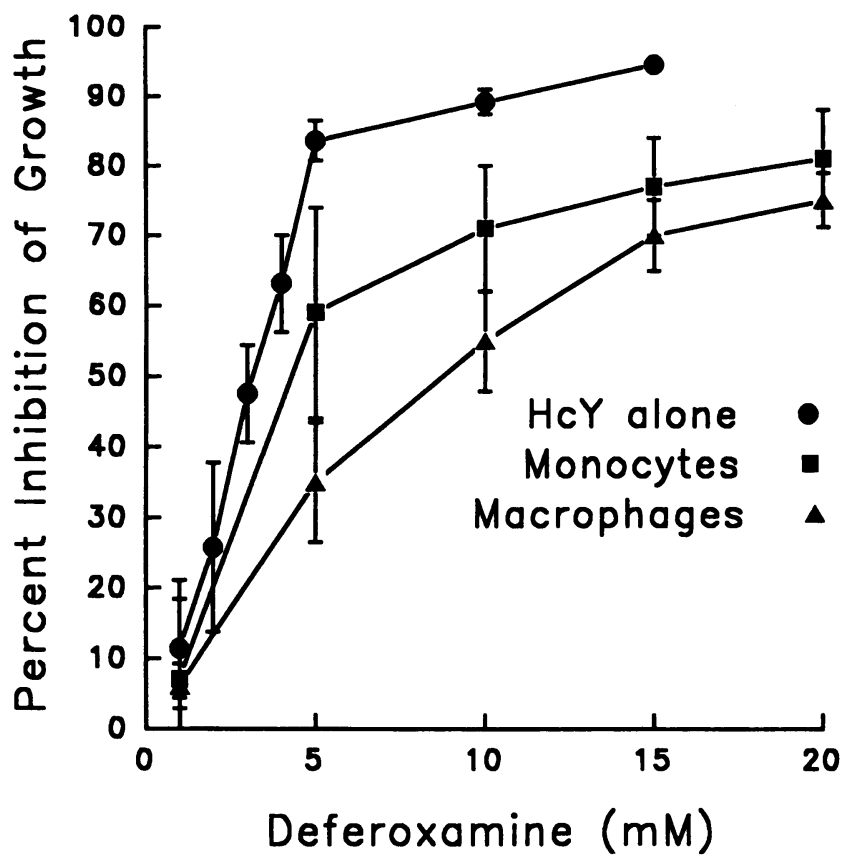

Figure 1. Deferoxamine inhibits the growth of Hc yeasts in human monocyte $/ \mathbf{M} \phi$ in a concentration-dependent manner. Monocyte $/ \mathbf{M} \phi$ were adhered in the wells of a 96-well tissue culture plate and then were infected with $5 \times 10^{3} \mathrm{Hc}$ yeasts. After allowing $1 \mathrm{~h}$ for phagocytosis, the cells were cultured for $24 \mathrm{~h}$ in medium alone or in medium containing varying concentrations of deferoxamine. Alternatively, yeasts were cultured extracellularly for $24 \mathrm{~h}$ in medium alone or in medium containing deferoxamine. All wells were pulsed for $24 \mathrm{~h}$ with $\left[{ }^{3} \mathrm{H}\right]$ leucine and were harvested as described in Methods. The data are the mean \pm SEM of eight experiments with $\mathrm{M} \phi$, five experiments with monocytes, and seven experiments in tissue culture medium.

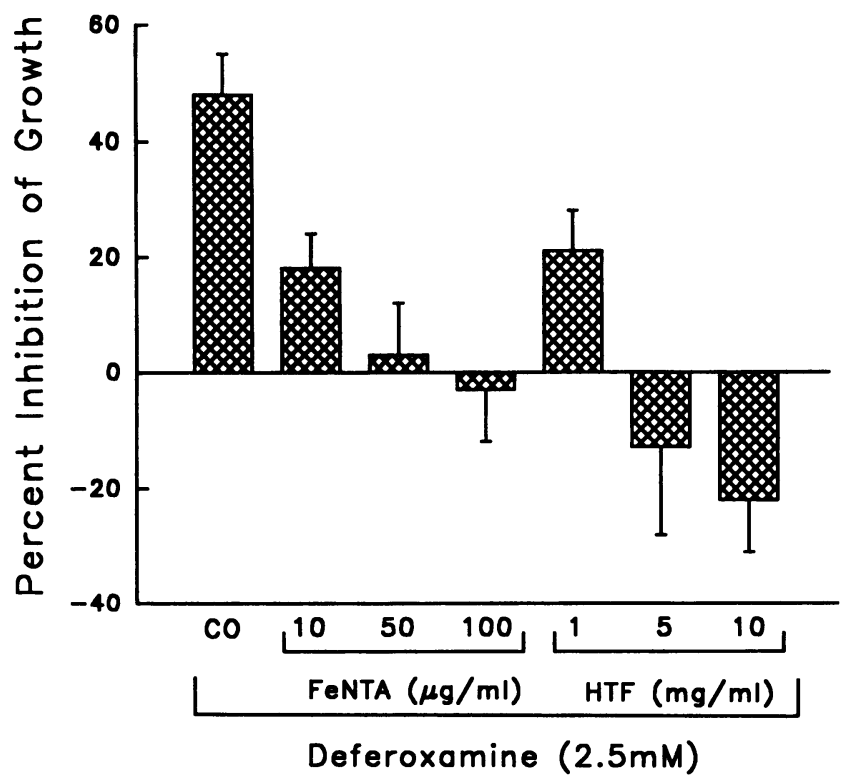

Figure 2. Reversal of deferoxamine-mediated inhibition of the intracellular growth of $\mathrm{Hc}$ yeasts by holotransferrin and FeNTA. M $\phi$ were infected with $5 \times 10^{3} \mathrm{Hc}$ yeasts and then were cultured for $24 \mathrm{~h}$ in medium, medium containing $2.5 \mathrm{mM}$ deferoxamine $(\mathrm{CO})$, or medium containing deferoxamine and varying concentrations of holotransferrin $(H T F)$ or FeNTA. The data are the mean \pm SEM of six experiments.

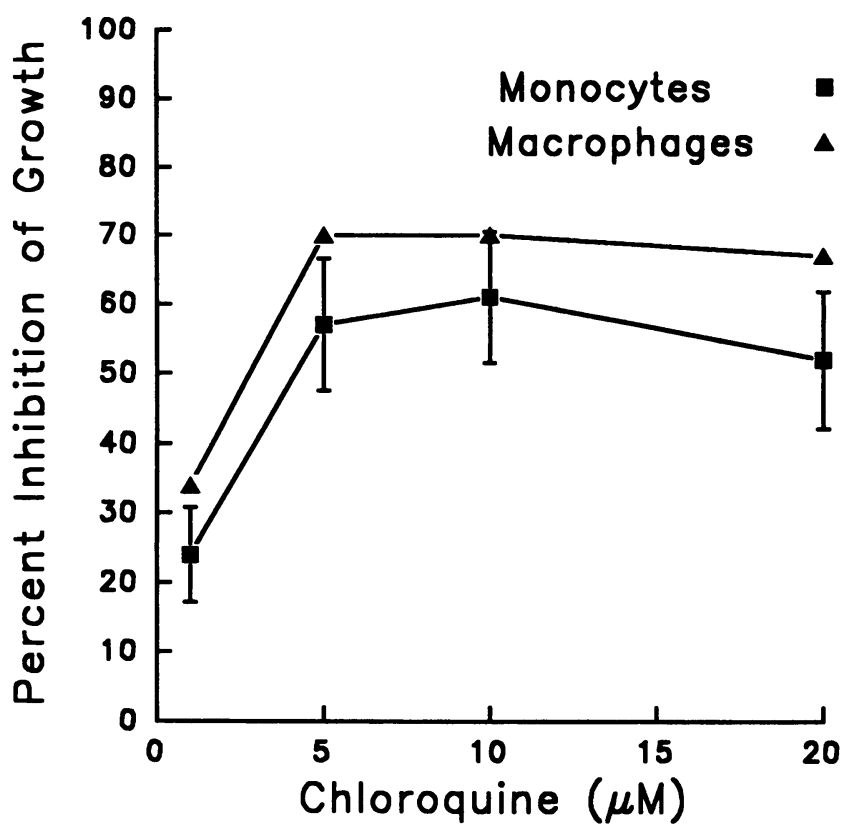

Figure 3. Chloroquine inhibits the growth of Hc yeasts in human monocyte $/ \mathbf{M} \phi$ in a concentration-dependent manner. Monocyte/M $\phi$ were infected with $5 \times 10^{3} \mathrm{Hc}$ yeasts and were cultured for $24 \mathrm{~h}$ in medium alone or in medium containing increasing concentrations of chloroquine. The data presented are the mean \pm SEM of seven experiments.

control M $\phi$. NTA $(100 \mu \mathrm{g} / \mathrm{ml})$ and apotransferrin $(10 \mathrm{mg} / \mathrm{ml})$ did not reverse deferoxamine-mediated inhibition of the growth of yeasts (data not shown).

Chloroquine inhibits the intracellular growth of $\mathrm{Hc}$ yeasts in human monocytes and $M \phi$. The intracellular transport and mobilization of iron are dependent on an acidic environment in endocytic vesicles and lysosomes $(17,18)$. Chloroquine is a

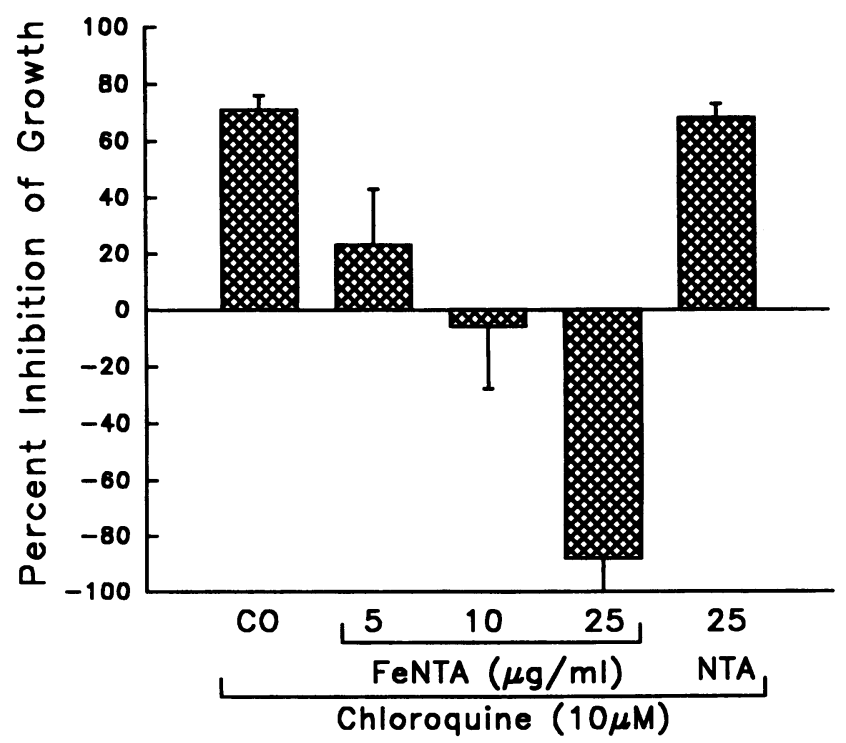

Figure 4. Reversal of chloroquine-mediated inhibition of the intracellular growth of Hc yeasts by FeNTA but not by NTA. M $\phi$ were infected with $5 \times 10^{3} \mathrm{Hc}$ yeasts and then were cultured for $24 \mathrm{~h}$ in medium alone, in medium containing $10 \mu \mathrm{M}$ chloroquine $(\mathrm{CO})$, or in medium containing chloroquine and FeNTA or NTA. The data are the mean \pm SEM of eight experiments. 


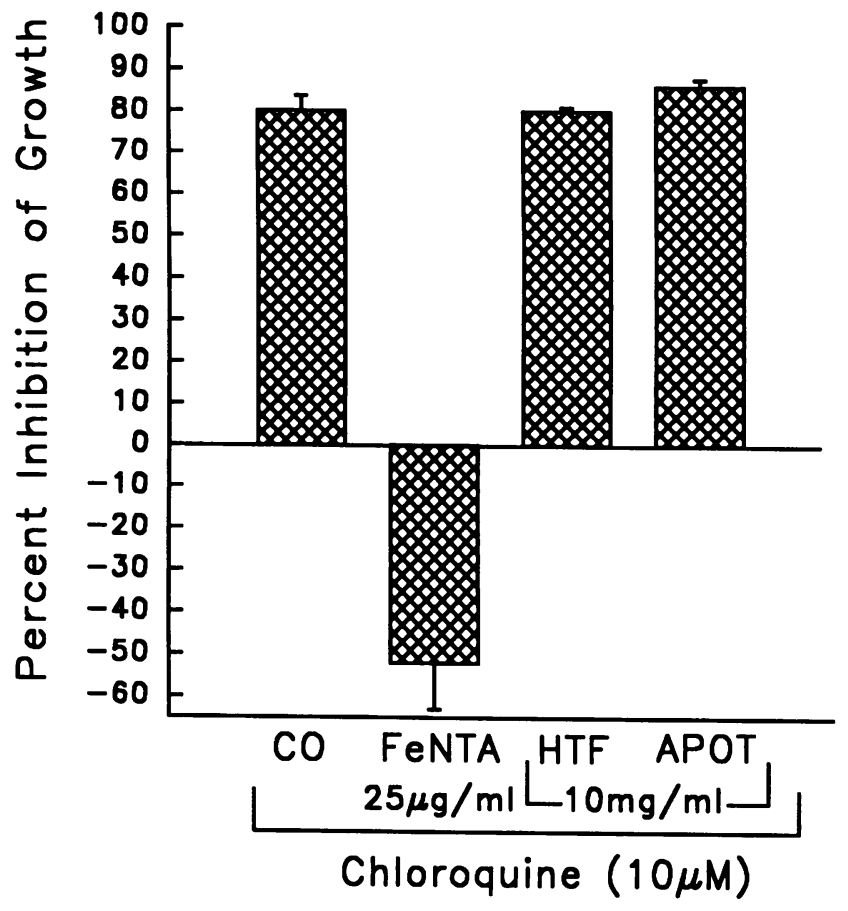

Figure 5. Reversal of chloroquine-mediated inhibition of the intracellular growth of Hc yeasts by FeNTA but not by holotransferrin or apotransferrin. $\mathrm{M} \phi$ were infected with $5 \times 10^{3} \mathrm{Hc}$ yeasts and then were cultured for $24 \mathrm{~h}$ in medium alone, in medium containing 10 $\mu \mathrm{M}$ chloroquine $(\mathrm{CO})$, or in medium containing chloroquine and FeNTA, holotransferrin $(H T F)$, or apotransferrin $(A P O T)$. The data are the mean \pm SEM of six experiments.

diprotic weak base that raises the $\mathrm{pH}$ of endocytic vesicles and lysosomes in eukaryotic cells (19). It therefore prevents the release of iron from transferrin in a variety of cell types including $\mathbf{M} \phi(19-22)$. Byrd and Horwitz (13) have shown that chloroquine inhibits the intracellular growth of $L$. pneumophila in human monocytes and that the effect of chloroquine is reversed by FeNTA, a compound in which iron remains soluble at neutral to alkaline $\mathrm{pH}(23)$.

Since the multiplication of $\mathrm{Hc}$ yeasts in human $\mathrm{M} \phi$ is iron dependent, we tested the hypothesis that chloroquine, by virtue of its capacity to interfere with iron metabolism, would inhibit the intracellular growth of $\mathrm{Hc}$ yeasts in a manner similar to that reported for L. pneumophila (13).

Monocyte and $\mathrm{M} \phi$ monolayers were infected with $\mathrm{Hc}$ yeasts and then were cultured for $24 \mathrm{~h}$ in medium alone or

Table I. Chloroquine Induces Killing of Hc Yeasts by Human Macrophages

\begin{tabular}{ccc}
\hline $\begin{array}{c}\text { Time after } \\
\text { infection }\end{array}$ & Chloroquine & Infected M $\phi$ \\
\hline$h$ & $\mu M$ & $\%$ \\
1 & None & $30 \pm 9.0^{*}$ \\
24 & None & $34 \pm 9.4$ \\
24 & 1 & $23 \pm 7.7$ \\
24 & 5 & $4.8 \pm 1.9$ \\
24 & 10 & $5.0 \pm 2.2$ \\
\hline
\end{tabular}

\footnotetext{
* Mean $\pm \operatorname{SEM}(n=6)$.
}

Table II. Treatment of Mice with Chloroquine Decreases the Growth of Hc Yeasts in Liver and Spleen

\begin{tabular}{|c|c|c|c|c|}
\hline \multirow[b]{2}{*}{ Treatment } & \multicolumn{2}{|c|}{ Spleen* } & \multicolumn{2}{|c|}{ Liver* } \\
\hline & Weight & $\mathrm{CFU}\left(\times 10^{6}\right)$ & Weight & $\mathrm{CFU}\left(\times 10^{6}\right)$ \\
\hline & $g$ & & $g$ & \\
\hline $\begin{array}{l}\text { RPMI } \\
\text { Chloroquine }\end{array}$ & $0.33 \pm 0.02$ & $1.70 \pm 0.12$ & $2.01 \pm 0.12$ & $12.1 \pm 2.30$ \\
\hline $120 \mathrm{mg} / \mathrm{kg}$ & $0.18 \pm 0.03$ & $0.19 \pm 0.07^{\ddagger}$ & $1.68 \pm 0.30$ & $0.83 \pm 0.46^{\ddagger}$ \\
\hline
\end{tabular}

${ }^{*}$ Mean $\pm \mathrm{SD}$ (seven mice per group). ${ }^{\ddagger} P<0.01$ compared with controls.

medium containing increasing concentrations of chloroquine. Culture of infected monocyte/M $\phi$ with chloroquine suppressed the intracellular growth of yeasts in a dose-dependent fashion with maximum inhibition at 5-10 $\mu \mathrm{M}$ chloroquine (Fig. 3). To exclude the possibility that chloroquine was directly toxic to yeasts, yeasts were cultured in tissue culture medium in the presence or absence of chloroquine. At a concentration of $20 \mu \mathrm{M}$, chloroquine inhibited the growth of yeasts by only $8.7 \pm 4.3 \%$ (mean \pm SEM, $n=3$ ).

FeNTA reverses chloroquine-mediated inhibition of the intracellular growth of Hc yeasts. We next sought to determine if chloroquine suppressed the intracellular growth of $\mathrm{Hc}$ yeasts by limiting the availability of iron. Hc yeast-infected $\mathrm{M} \phi$ were incubated with $10 \mu \mathrm{M}$ chloroquine in the absence and presence of varying concentration of FeNTA (23). Addition of FeNTA to Hc-infected $\mathrm{M} \phi$ treated with chloroquine reversed the inhibition of yeast growth by chloroquine (Fig. 4). At $25 \mu \mathrm{g} / \mathrm{ml}$, the highest concentration of FeNTA tested, intracellular yeasts grew more rapidly than in untreated control M $\phi$. NTA at 25 $\mu \mathrm{g} / \mathrm{ml}$ did not block the effect of chloroquine. These results establish that the effect of FeNTA was mediated by iron and not by NTA.

FeNTA, but not holotransferrin, reverses chloroquine-mediated inhibition of the intracellular growth of Hc yeasts. By increasing the $\mathrm{pH}$ in endocytic vesicles, chloroquine prevents the release of iron from endocytized transferrin, a process that is dependent on endocytic vesicle acidification $(17,18)$. Thus, the addition of iron transferrin to chloroquine-treated $\mathrm{M} \phi$ would not be expected to reverse the growth inhibitory effect of chloroquine. To test this hypothesis, Hc-infected $\mathrm{M} \phi$ were cultured for $24 \mathrm{~h}$ in the presence of $10 \mu \mathrm{M}$ chloroquine alone or with chloroquine and $25 \mu \mathrm{g} / \mathrm{ml}$ of FeNTA, $10 \mathrm{mg} / \mathrm{ml}$ of holotransferrin, or $10 \mathrm{mg} / \mathrm{ml}$ of apotransferrin. FeNTA completely reversed the effect of chloroquine, whereas holotransferrin and apotransferrin did not (Fig. 5).

Chloroquine induces intracellular killing of Hc yeasts by human $M \phi$. In the presence of cycloheximide, human $\mathbf{M} \phi \mathrm{di}-$ gest intracellular Hc yeasts (4), suggesting that inhibition of yeast cell metabolism renders Hc susceptible to killing by $\mathbf{M} \phi$. Chloroquine clearly disrupts yeast cell metabolism by restricting the availability of intracellular iron. Since the radiometric assay does not distinguish between killing and growth inhibition, we quantified the killing of $\mathrm{Hc}$ yeasts in chloroquinetreated $\mathbf{M} \phi$ by counting the percentage of infected $\mathbf{M} \phi$ by phase-contrast microscopy (4).

$\mathrm{M} \phi$ were infected with Hc yeasts and were either fixed immediately ( $1 \mathrm{~h}$ after infection) or were cultured for $24 \mathrm{~h}$ at 


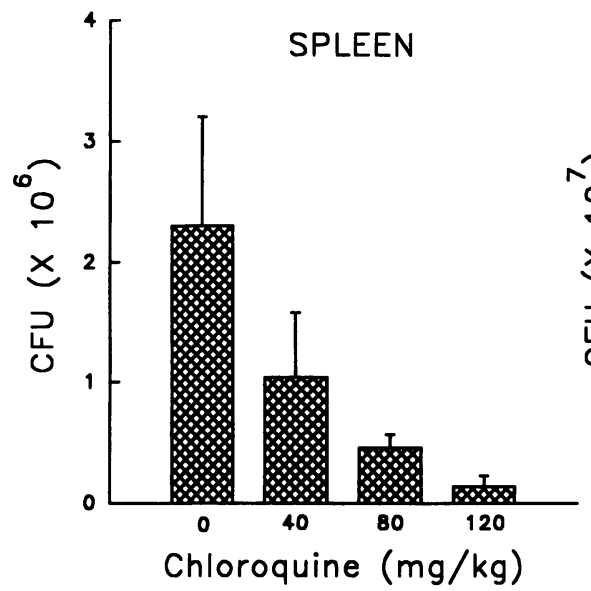

Chloroquine $(\mathrm{mg} / \mathrm{kg})$

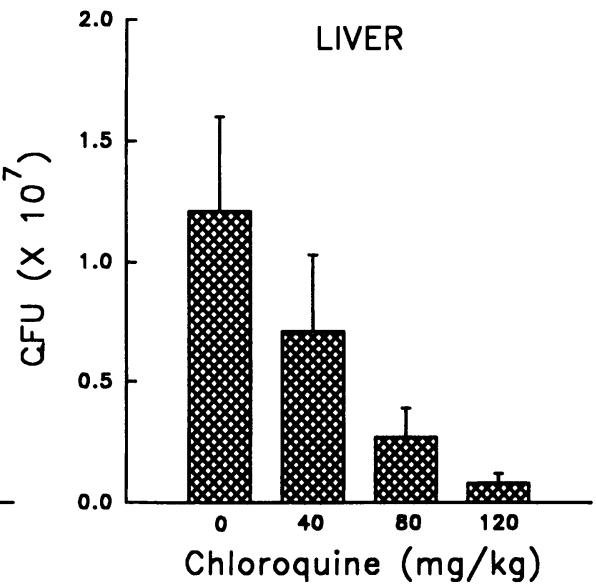

Figure 6. Chloroquine treatment reduces the number of yeasts in the spleens and livers of Hc-infected mice in a dose-dependent manner. Mice were injected intravenously with $1 \times 10^{6} \mathrm{Hc}$ yeasts. On the same day, mice were given chloroquine 40,80 , or $120 \mathrm{mg} / \mathrm{kg}$ intraperitoneally and were treated daily for $6 \mathrm{~d}$. The data are the mean \pm SD for six mice in each group. Each dose of chloroquine significantly reduced $\mathrm{CFU}$ in spleens and livers as compared with Hc-infected controls: $P<0.05$ for $40 \mathrm{mg}$ / $\mathrm{kg} ; P<0.01$ for 80 and $120 \mathrm{mg} / \mathrm{kg}$. $37^{\circ} \mathrm{C}$ in medium alone or in medium containing varying concentrations of chloroquine. After $1 \mathrm{~h}$ of phagocytosis, $30 \%$ of $\mathrm{M} \phi$ were infected with one or more yeasts (Table I). After $24 \mathrm{~h}$ of culture in medium alone, $34 \%$ of $\mathrm{M} \phi$ were infected, and the average number of yeasts/ $\mathrm{M} \phi$ had increased from 2.7 to 3.6. However, culture of infected $\mathrm{M} \phi$ in the presence of 5-10 $\mu \mathrm{M}$ chloroquine reduced the percentage of infected $M \phi$ to $5 \%$ and the number of yeasts $/ \mathrm{M} \phi$ to 1.7 at $24 \mathrm{~h}$. Thus, in the presence of chloroquine, $\mathbf{M} \phi$ killed and digested Hc yeasts.

Treatment of $\mathrm{Hc}$-infected mice with chloroquine reduces the number of organisms in the spleen and liver. These findings suggested that chloroquine might be effective against $\mathrm{Hc}$ in vivo. Therefore, C57BL/6 mice were injected intravenously with $1 \times 10^{6} \mathrm{Hc}$ yeasts. $8 \mathrm{~h}$ later, chloroquine, $120 \mathrm{mg} / \mathrm{kg}$, was given intraperitoneally and continued daily thereafter. Mice were killed on day 7. Chloroquine was well tolerated, and the mice maintained their normal eating and drinking habits.

Treatment of mice with chloroquine reduced the number of organisms in the spleens and livers by 9 - and 15-fold, respectively $(P<0.01$ as compared with controls, Wilcoxon rank sum test) ( Table II). In subsequent trials, groups of mice were given 40,80 , or $120 \mathrm{mg} / \mathrm{kg}$ of chloroquine $8 \mathrm{~h}$ after intravenous injection of yeasts and daily for $6 \mathrm{~d}$. Each dose of chloroquine significantly reduced CFU in spleens and livers as compared with Hc-infected controls (Fig. 6).

Additional studies were performed to determine the efficacy of chloroquine when administrated later in the course of

Table III. Chloroquine Is Efficacious When Given during Active Hc Infection

\begin{tabular}{lcccc}
\hline & \multirow{2}{*}{$\begin{array}{c}\text { Days of } \\
\text { infection } \\
\text { before }\end{array}$} & \multicolumn{2}{c}{ CFU $\left(10^{6}\right)^{*}$} \\
\cline { 3 - 4 } \multicolumn{1}{c}{ Treatment } & treatment & & Spleen & Liver \\
\hline RPMI & $0(7)^{\ddagger}$ & $2.18 \pm 0.99$ & $15.9 \pm 4.3$ \\
Chloroquine $120 \mathrm{mg} / \mathrm{kg}$ & $5(2)$ & $1.06 \pm 0.35$ & $13.3 \pm 2.2$ \\
& $3(4)$ & $0.40 \pm 0.29^{\S}$ & $7.7 \pm 4.8^{\S}$ \\
& $0(7)$ & $0.13 \pm 0.09^{\prime \prime}$ & $2.3 \pm 1.3^{\prime \prime}$
\end{tabular}

${ }^{*}$ Mean \pm SD (six mice per group). ${ }^{\ddagger}$ Numbers in parentheses represent the number of days of treatment. ${ }^{\S} P<0.05$; $" P<0.01$. infection. Mice were injected intravenously with $1 \times 10^{6} \mathrm{Hc}$ yeasts, and therapy was initiated with chloroquine $(120 \mathrm{mg} /$ $\mathrm{kg}$ ) at $8 \mathrm{~h}$, on day 3 or on day 5 after inoculation. Treatment was continued daily until mice were killed on day 7 . After $7 \mathrm{~d}$ of treatment, the CFU in spleens and livers were reduced 17 - and 7 -fold, respectively $(P<0.01)$ (Table III). After $4 \mathrm{~d}$ of treatment, CFU in the spleens and livers were reduced by five- and twofold, respectively $(P<0.05)$. In mice in which Hc were allowed to proliferate for $4 \mathrm{~d}$ before receiving only $2 \mathrm{~d}$ of chloroquine therapy, the number of organisms in the spleens still was reduced by $50 \%$. Thus, the efficacy of chloroquine correlated with the length of treatment.

Treatment of $\mathrm{Hc}$-infected mice with chloroquine protects them from a lethal inoculum of $\mathrm{Hc}$ yeasts. Although chloroquine was therapeutic against a sublethal challenge, we sought to determine if it could protect mice from a lethal inoculum of yeasts. 20 mice were given $1.5 \times 10^{7}$ yeasts. $8 \mathrm{~h}$ after inoculation, groups of 10 mice were treated either with $120 \mathrm{mg} / \mathrm{kg}$ of chloroquine or with RPMI. Chloroquine was continued daily

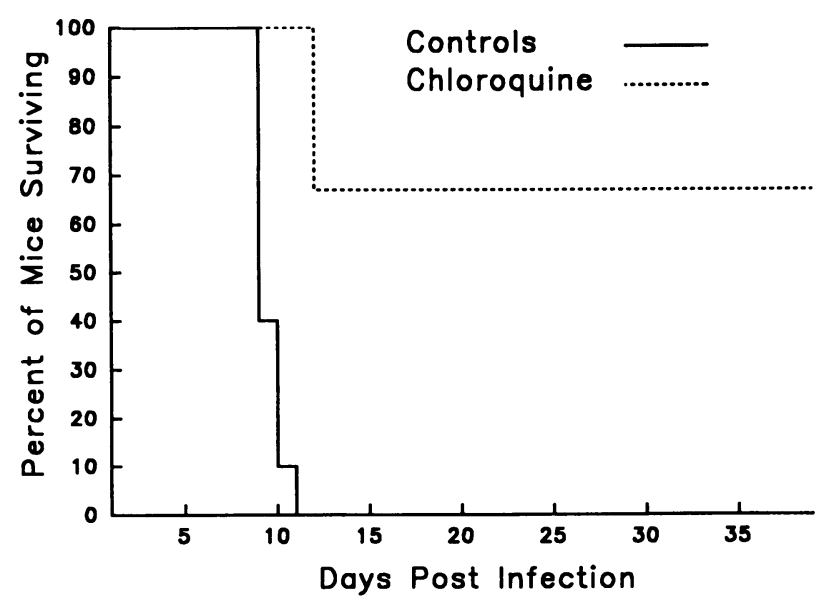

Figure 7. Chloroquine protects mice from a lethal inoculum of $\mathrm{Hc}$ yeasts. 20 mice were given a lethal inoculum of $\mathrm{Hc}$ yeasts $\left(1.5 \times 10^{7}\right)$. Groups of 10 mice were given chloroquine intraperitoneally ( 120 $\mathrm{mg} / \mathrm{kg}$ ) or RPMI on the day of infection. Treatment was continued for $10 \mathrm{~d}$. Mice were observed for $\mathbf{4 0} \mathrm{d}$. The survival of the chloroquine-treated mice was significantly different from the controls $(P$ $=0.009$, Fisher's exact test ). 
for $10 \mathrm{~d}$. After $11 \mathrm{~d}$, all 10 mice in the control group had died (Fig. 7). One mouse in the chloroquine-treated group died on day 3 from intraperitoneal hemorrhage secondary to trauma from injection. Of the remaining nine mice, three died on day 13, and six mice survived until day 40 when the experiment was terminated (Fig. 7). Extensive cultures of the spleens and livers of the surviving mice yielded no growth of $\mathrm{Hc}$.

\section{Discussion}

Iron is an essential element for the growth and metabolism of almost all living cells (24). Iron functions in the transport and storage of oxygen and as a catalyst in electron transport processes. Thus, the acquisition of iron from the host is critical for pathogenic microorganisms to successfully establish infection. Conversely, restriction of the availability of iron to invading microbes would be advantageous to the host (25-27).

Despite the abundance of iron in the mammalian host, free iron is very limited. Most of the iron is located intracellularly, making acquisition of iron by extracellular pathogens difficult. The small amount of iron that is in body fluids is bound by the high affinity iron-binding glycoproteins transferrin and lactoferrin (28). These host proteins may provide nonspecific nutritional immunity by restricting infective microbial growth through the deprivation of iron (25-27). Thus, addition of unsaturated transferrin to culture medium inhibits the growth of Hc (29) and other fungi, including Candida albicans (30), Candida glabrata (31), and Cryptococcus neoformans (32).

During infection, iron availability is restricted further by the induction of hypoferremia, a manifestation of the acutephase response. The decrease in free iron is associated with an increase in serum transferrin and lactoferrin $(26,33-35)$. The major mechanism whereby iron decreases in serum and concomitantly accumulates in the spleen and liver is by inhibition of the release of iron from $\mathbf{M} \phi$, which prevents its normal recycling to transferrin (36). $M \phi$ also initiate the synthesis of the iron storage protein ferritin, which enables $\mathbf{M} \phi$ to continue to acquire iron from ingested senescent erythrocytes (36). Paradoxically, this response to infection may enhance the pathogenicity of intracellular parasites by providing them with sufficient iron to multiply successfully within $\mathbf{M} \phi$.

This study demonstrates that the availability of intracellular iron is critical for Hc yeasts to survive and multiply within the phagolysosomes of human $\mathrm{M} \phi$. Culture of Hc-infected human $\mathbf{M} \phi$ with the iron chelator deferoxamine inhibited the intracellular growth of Hc yeasts. The effect of deferoxamine was reversed by FeNTA and holotransferrin but not by NTA or apotransferrin.

In $\mathbf{M} \phi$, mobilization of iron from transferrin and ferritin is dependent upon an acidic environment in endocytic vesicles and lysosomes $(17,18,37-39)$. After the transferrin receptor binds diferric transferrin, the receptor-ligand complex is endocytized, and the endocytic vesicle is acidified. As the $\mathrm{pH}$ decreases from 7 to 6 , ferric ions dissociate from transferrin and are distributed intracellularly. At pH 6, one of two ferric ions bound to transferrin dissociates, resulting in $50 \%$ iron saturation of transferrin. When the $\mathrm{pH}$ decreases to 5 , the second ferric ion dissociates $(40,41)$. The unsaturated apotransferrin remains bound to the transferrin receptor and is recycled to the cell surface (18). In contrast, iron that is bound to ferritin is released to the intermediate pool of the cell after ferritin is degraded in lysosomes $(37,38)$. Chloroquine prevents the release of ferric ions from saturated iron transferrin (19-22) and may prevent degradation of ferritin by acid proteases (39) by virtue of its ability to raise endocytic and lysosomal $\mathrm{pH}$.

Coculture of $\mathrm{Hc}$-infected human $\mathrm{M} \phi$ with 5-10 $\mu \mathrm{M}$ chloroquine induced $\mathrm{M} \phi$ to kill $\mathrm{Hc}$ yeasts. The effect of chloroquine was reversed by FeNTA, an iron compound that is soluble in the neutral to alkaline $\mathrm{pH}$ range (23), but not by holotransferrin, which requires an acid $\mathrm{pH}$ to release ferric ions $(40,41)$. NTA did not reverse the effect of chloroquine. Thus, iron, and not the parent compound, restored the capacity of $\mathrm{Hc}$ to multiply in the presence of chloroquine.

In a recent study, Eissenberg and Goldman (42) demonstrated that phagosomes of P388D1 mouse $\mathrm{M} \phi$ cell line containing viable $\mathrm{Hc}$ yeasts have a $\mathrm{pH}$ of $\sim 6.0-6.5$. In contrast, vesicles containing zymosan or methanol-fixed yeasts maintained a pH of 5.5 over a period of several hours. These data, in conjunction with the present experiments, suggest a strategy by which $\mathrm{Hc}$ yeasts may survive and multiply within $\mathrm{M} \phi$ phagolysosomes. By maintaining a vacuolar $\mathrm{pH}$ of about 6 , lysosomal acid hydrolases that are detrimental to $\mathrm{Hc}$ presumably are inactivated. However, at pH 6, one molecule of iron would be released from transferrin and would be available to the yeasts for cellular metabolism. The addition of chloroquine to Hc-infected $\mathrm{M} \phi$ upsets this balance by raising the $\mathrm{pH}$ above 6.5. Thus, iron is not available to the yeasts, and, subsequently, they are killed.

Several experiments demonstrated unequivocally that our in vitro findings were applicable in vivo. First, treatment with chloroquine reduced the number of organisms in the spleens and livers of Hc-infected mice in a dose-dependent manner. Second, the effectiveness of chloroquine in reducing the numbers of yeasts in the spleens and livers correlated with the length of therapy. Third, $67 \%$ of mice given chloroquine were protected from a lethal inoculum of Hc yeasts, whereas all untreated mice were dead by day 11 .

These studies confirm and extend the studies of Byrd and Horwitz (13), who demonstrated that chloroquine inhibits the growth of $L$. pneumophila in human monocytes by restricting the availability of intracellular iron. Likewise, chloroquine inhibits the growth of Mycobacterium tuberculosis in human $\mathbf{M} \phi$ and also significantly enhances the effectiveness of isoniazid and pyrazinamide against intracellular $M$. tuberculosis (43). The mechanism by which chloroquine inhibits the intracellular growth of $M$. tuberculosis has not been explored. However, it seems likely that the effect of chloroquine may be mediated by restriction of the availability of intracellular iron.

Finally, it has been shown by other investigators that the ability of resident or elicited murine PM to kill Listeria monocytogenes (44) and to inhibit the replication of Trypanosoma cruzi amastigotes (45) also is dependent on the concentration of intracellular iron. Culture of $T$. cruzi-infected PM in the presence of desferrioxamine reduces the rate of amastigote replication. Furthermore, $T$. cruzi-infected mice fed iron dextran to increase intracellular iron stores have an increased mortality rate. Conversely, depletion of host iron stores with desferroxamine and an iron-deficient diet reduce the pathogenicity of infection with $T$. cruzi (46).

Chloroquine is absorbed rapidly and completely from the gastrointestinal tract and achieves concentrations in the liver, 
spleen, kidney, and lungs that are 200-700 times those obtained in serum (47). Although excessive dosage can cause ocular toxicity, dosages of $4 \mathrm{mg} / \mathrm{kg}$ per day of chloroquine and $6.5 \mathrm{mg} / \mathrm{kg}$ per day of hydroxychloroquine have been found to be clinically safe in the management of rheumatoid arthritis and other connective tissue diseases $(48,49)$. At these dosages, serum concentrations of chloroquine are 1-1.5 $\mu \mathrm{M}$, and a concentration of $300-1,050 \mu \mathrm{M}$ might be obtained in the liver and spleen $(48,49)$. Thus, chloroquine, hydroxychloroquine, or other lysosomotropic agents that raise intraphagosomal $\mathrm{pH}$ may be safe and effective therapeutic agents for the treatment of active histoplasmosis and also may be useful in preventing relapse of histoplasmosis in patients with acquired immunodeficiency syndromes. In addition, chloroquine alone or in combination with other drugs may prove to be effective in augmenting the host's capacity to destroy other intracellular parasites that require iron for survival $(13,43-46)$. A potential advantage of chloroquine, or a similar acting drug, as an antimicrobial agent is that it does not act directly on the invading pathogen, but rather restricts the availability of intracellular iron. Therefore, the potential for microorganisms to become resistant to its effects may be limited.

\section{Acknowledgments}

We thank Ward Bullock for critical review of the manuscript.

This work was supported in part by National Institutes of Health grants $\mathrm{AI}-28392, \mathrm{AI}-23985$, and $\mathrm{AI}-23017$.

\section{References}

1. Bullock, W. E., and S. D. Wright. 1987. Role of adherence-promoting receptors, CR3, LFA-1, and p150,95, in binding of Histoplasma capsulatum by human macrophages. J. Exp. Med. 165:195-210.

2. Newman, S. L., C. Bucher, J. Rhodes, and W. E. Bullock. 1990. Phagocytosis of Histoplasma capsulatum yeasts and microconidia by human cultured macrophages and alveolar macrophages. Cellular cytoskeleton requirement for attachment and ingestion. J. Clin. Invest. 85:223-230.

3. Schnur, R. A., and S. L. Newman. 1990. The respiratory burst response to Histoplasma capsulatum by human neutrophils. Evidence for intracellular trapping of superoxide anion. J. Immunol. 144:4765-4772.

4. Newman, S. L., L. Gootee, R. Morris, and W. E. Bullock. 1992. Digestion of Histoplasma capsulatum yeasts by human macrophages. J. Immunol. 149:574-580.

5. Fleischmann, J., B. Wu-Hsieh, and D. H. Howard. 1990. The intracellular fate of Histoplasma capsulatum in human macrophages is unaffected by recombinant human interferon- $\gamma$. J. Infect. Dis. 161:143-145.

6. Newman, S. L., L. Gootee, C. Bucher, and W. E. Bullock. 1991. Inhibition of intracellular growth of Histoplasma capsulatum yeasts cells by cytokine-activated human monocytes and macrophages. Infect. Immun. 59:737-741.

7. Newman, S. L., and L. Gootee. 1992. Colony-stimulating factors activate human macrophages to inhibit the intracellular growth of Histoplasma capsulatum yeasts. Infect. Immun. 60:4593-4597.

8. Lane, T. E., B. A. Wu-Hsieh, and D. H. Howard. 1991. Iron limitation and the gamma interferon-mediated antihistoplasma state of murine macrophages. Infect. Immun. 59:2274-2278.

9. Lane, T. E., B. A. Wu-Hsieh, and D. H. Howard. 1993. Gamma interferon cooperates with lipopolysaccharide to activate mouse splenic macrophages to an antihistoplasma state. Infect Immun. 61:1468-1473.

10. Byrd, T. F., and M. A. Horwitz. 1989. Interferon gamma-activated human monocytes downregulate transferrin receptors and inhibit the intracellular multiplication of Legionella pneumophila by limiting the availability of iron. J. Clin. Invest. 83:1457-1465.

11. Byrd, T. F., and M. A. Horwitz. 1991. Lactoferrin inhibits or promotes Legionella pneumophila intracellular multiplication in nonactivated and interferon gamma-activated human monocytes depending upon its degree of iron saturation. Iron-lactoferrin and nonphysiologic iron chelates reverse monocyte activation against Legionella pneuomophila. J. Clin. Invest. 88:1103-1112.

12. Brummer, E., N. Kurita, S. Yoshida, K. Nishimura, and M. Miyaji. 1991. Killing of Histoplasma capsulatum by $\gamma$-interferon-activated human monocytederived macrophages: evidence for a superoxide anion-dependent mechanism. $J$. Med. Microbiol. 35:29-34.

13. Byrd, T. F., and M. A. Horwitz. 1991. Chloroquine inhibits the intracellular multiplication of Legionella pneumophila by limiting the availability of iron. A potential new mechanism for the therapeutic effect of chloroquine against intracellular pathogens. J. Clin. Invest. 88:351-357.

14. Worsham, P., and W. E. Goldman. 1988. Quantitative plating of Histoplasma capsulatum without addition of conditioned medium or siderophores. $J$. Med. Vet. Mycol. 26:137-143.

15. Newman, S. L., R. A. Musson, and P. M. Henson. 1980. Development of functional complement receptors during in vitro maturation of human monocytes into macrophages. J. Immunol. 125:2236-2244.

16. Gomez, F. J., A. M. Gomez, and G. S. Deepe, Jr. 1991. Protective efficacy of a 62-kilodalton antigen, HIS-62, from the cell wall and cell membrane of Histoplasma capsulatum yeast cells. Infect Immun. 59:4459-4464.

17. Jacobs, A. 1977. Low molecular weight intracellular iron transport compounds. Blood. 50:433-439.

18. Dautry-Varsat, A., A. Ciechanover, and H. F. Lodish. 1983. pH and the recycling of transferrin during receptor-mediated endocytosis. Proc. Natl. Acad. Sci. USA. 80:2258-2262.

19. Krogstad, D. J., and P. H. Schlesinger. 1987. Acid-vesicle function, intracellular pathogens, and the action of chloroquine against Plasmodium falciparum. N. Engl. J. Med. 317:542-549.

20. Swaiman, K. F., and V. L. Machen. 1986. Chloroquine reduces neuronal and glial iron uptake. J. Neurochem. 46:652-654.

21. Baynes, R., G. Bukofzer, T. Bothwell, W. Bezwoda, and B. Macfarlane. 1987. Transferrin receptors and transferrin iron uptake by cultured human blood monocytes. Eur. J. Cell Biol. 43:372-376.

22. Forsbeck, K., and K. Nilsson. 1983. Iron metabolism of established human hematopoietic cell lines in vitro. Exp. Cell Res. 144:323-332.

23. Bates, G. W., and J. Wernicke. 1971. The kinetics and mechanism of iron (III) exchange between chelates and transferrin. J. Biol. Chem. 246:3679-3686. 24. Neilands, J. B. 1972. Evolution of biological iron binding centers. Struct. Bonding. 11:145-170.

25. Finkelstein, R. A., C. V. Sciortino, and M. A. McIntosh. 1983. Role of iron in microbe-host interactions. Rev. Infect. Dis. 5:S759-S777.

26. Weinberg, E. D. 1984. Iron withholding: a defense against infection and neoplasia. Physiol. Rev. 64:65-102.

27. Litwin, C. M., and S. B. Calderwood. 1993. Role of iron in regulation of virulence genes. Clin. Microbiol. Rev. 6:137-149.

28. Aisen, P., and A. Leibman. 1972. Lactoferrin and transferrin: a comparative study. Biochim. Biophys. Acta. 257:314-323.

29. Sutcliffe, M. C., A. M. Savage, and R. H. Alford. 1980. Transferrin-dependent growth inhibition of yeast-phase Histoplasma capsulatum by human serum and lymph. J. Infect. Dis. 142:209-219.

30. Caroline, L., C. L. Taschdjian, P. J. Kozinn, and A. L. Schade. 1964. Reversal of serum fungistasis by addition of iron. J. Invest. Dermatol. 42:415419.

31. Otto, V., and D. H. Howard. 1976. Further studies on the intracellular behavior of Torulopsis glabrata. Infect. Immun. 14:433-438.

32. Hendry, A. T., and A. Bakerspigel. 1969. Factors affecting serum inhibited growth of Candida albicans and Cryptococcus neoformans. Sabouraudia. 7:219229.

33. Muller, D., M. L. Edwards, and D. W. Smith. 1983. Changes in iron and transferrin levels and body temperature in experimental airborne legionellosis. $J$. Infect. Dis. 147:302-307.

34. Van Snick, J. L., P. L. Masson, and J. F. Heremans. 1974. The involvement of lactoferrin in the hyposideremia of acute inflammation. J. Exp. Med. 140:1068-1084.

35. Wright, D. G., and J. I. Gallin. 1979. Secretory responses of human neutrophils: exocytosis of specific (secondary) granules by human neutrophils during adherence in vitro and during exudation in vivo. J. Immunol. 123:285-294.

36. Zahringer, J., B. S. Baliga, and H. N. Munro. 1976. Novel mechanism for translational control in regulation of ferritin synthesis by iron. Proc. Natl. Acad. Sci. USA. 73:857-861.

37. Laub, R., Y. Schneider, J. Octave, A. Trouet, and R. R. Crichton. 1985. Cellular pharmacology of deferrioxamine $B$ and derivatives in cultured rat hepatocytes in relation to iron mobilization. Biochem. Pharmacol. 34:1175-1183.

38. Starke, P. E., J. D. Gilbertson, and J. L. Farber. 1985. Lysosomal origin of the ferric iron required for cell killing by hydrogen peroxide. Biochem. Biophys. Res. Commun. 133:371-379.

39. Sibille, J., H. Kondo, and P. Aisen. 1989. Uptake of ferritin and iron bound to ferritin by rat hepatocytes: modulation by apotransferrin, iron chelators and chloroquine. Biochim. Biophys. Acta. 1010:204-208. 
40. Princiotto, J. V and E. J. Zapolski. 1975. Difference between the two iron binding sites of transferrin. Nature (Lond.). 255:87-88.

41. Lestas, A. N. 1976. The effect of pH upon human transferrin: selective labeling of the two iron-binding sites. Br. J. Haematol. 32:341-350.

42. Eissenberg, L. G., W. E. Goldman, and P. H. Schlesinger. 1993. Histo plasma capsulatum modulates the acidification of phagolysosomes. J. Exp. Med. 177:1605-1611.

43. Crowle, A. J., and M. H. May. 1990. Inhibition of tubercle bacilli in cultured human macrophages by chloroquine used alone and in combination with streptomycin, isoniazid, pyrazinamide, and two metabolites of vitamin $\mathrm{D}_{3}$. Antimicrob. Agents Chemother. 34:2217-2222.

44. Alford, C. E., T. E. King, Jr., and P. A. Campbell. 1991. Role of transferrin, transferrin receptors, and iron in macrophage listericidal activity. J. Exp. Med. 174:459-466.
45. Loo, V. G., and R. G. Lalonde. 1984. Role of iron in intracellular growth of Trypanosoma cruzi. Infect. Immun. 45:726-730.

46. Lalonde, R. G., and B. E. Holbein. 1984. Role of iron in Trypanosoma cruzi infection of mice. J. Clin. Invest. 73:470-476.

47. Marr, J. I. 1992. Antiparasitic agents. In Principles and Practice of Infectious Disease. G. L. Mandell, R. G. Douglas, and J. E. Bennett, editors. John Wiley \& Sons Inc., New York. 286-301.

48. McChesney, E. W., W. F. Banks, Jr., and J. P. McAuliff. 1962. Laboratory studies of the 4-aminoquinoline antimalarials. II. Plasma levels of chloroquine and hydroxychloroquine in man after various oral dosage regimens. Antibiot. Chemother. (Wash. DC). 12:583-594.

49. Mackenzie, A. H. 1983. Pharmacologic actions of 4-aminoquinoline compounds. Am. J. Med. 75:5-10. 